\title{
Modulation of the Startle Reflex during Brief and Sustained Exposure to Emotional Pictures
}

\author{
Aimee Mavratzakis ${ }^{1,2}$, Elaine Molloy ${ }^{1}$, Peter Walla ${ }^{1,2^{*}}$ \\ ${ }^{1}$ Faculty of Science and Information Technology, School of Psychology, University of Newcastle, \\ Callaghan, Australia \\ ${ }^{2}$ Centre for Translational Neuroscience and Mental Health Research, University of Newcastle, \\ Callaghan, Australia \\ Email: "peter.walla@newcastle.edu.au
}

Received December $6^{\text {th }}, 2012$; revised January $7^{\text {th }}, 2013$; accepted February $5^{\text {th }}, 2013$

\begin{abstract}
Copyright (C) 2013 Aimee Mavratzakis et al. This is an open access article distributed under the Creative Commons Attribution License, which permits unrestricted use, distribution, and reproduction in any medium, provided the original work is properly cited.
\end{abstract}

\begin{abstract}
Previous investigations using pictures to elicit an emotional response have shown that the startle reflex habituates over time due to decreased excitation in the obligatory startle processing pathway, an effect that is independent of emotion modulation aspects of the startle response. However, in some instances, startle magnitude has been selectively potentiated during sustained exposure to passively viewed unpleasant pictures. This study assessed startle modulation during brief, alternating and sustained exposure to emotional pictures. Self-reported ratings of emotion were collected online with picture viewing to determine if any change in startle magnitude was observable in explicit emotional responses. Self-reported ratings of pleasantness and arousal were no different across the brief and sustained picture presentations. However, a significant main effect (independent from emotion category) of presentation condition was found for startle magnitudes, showing that, contrary to previous research involving passive picture viewing, mean startle magnitudes during sustained exposure were reduced relative to brief exposure. These findings are likely the result of a general habituation of the startle reflex in the obligatory pathway. The findings are also discussed in terms of the effect of the concurrent emotion rating task, which may have differently affected the cumulative effects of emotion exposure compared to passive picture viewing.
\end{abstract}

Keywords: Emotion; Explicit; Implicit; Brief Exposure; Sustained Exposure

\section{Introduction}

The startle reflex is an indicator of the brains aversive motivational system via its structural connection to motivation systems at the synaptic level (Yeomans \& Frankland, 1995). It is a sudden involuntary movement involving the whole body (muscle contractions), and is thought to serve as a mechanism for quickly withdrawing the whole organism from harm. The reflex is activated in response to an intense unexpected stimulus with a rapid rising time and can be elicited via any of the senses (e.g. loud noise, flash of light, air puff). Reflexes are by definition automatic, however it is also known that activation patterns of reflexes vary as a function of psychological factors (Filion, Dawson, \& Schell, 1998).

Previous investigations of the neural circuitry influencing acoustic startle magnitude suggest input from two distinct circuits. The primary obligatory circuit projects from the cochlear nucleus to the spinal cord and the secondary modulatory circuit, which includes the amygdala connects with the primary obligatory circuit causing its modulation (Yeomans \& Frankland, 1995). The obligatory circuit is thought to be responsible for the early excitatory startle reactivity seen in initial startles, which rapidly habituates after about six trials (Blumenthal et al., 2005). Bradley and colleagues demonstrated habituation of the

\footnotetext{
${ }^{*}$ Corresponding author.
}

obligatory pathway in a study investigating startle responses to repeated presentations of the same unpleasant, pleasant and neutral pictures over time (Bradley, Lang, \& Cuthbert, 1993). They showed that while the overall strength of the reflex gradually reduced, blink magnitude continued to discriminate between negative and positive stimuli, which shows that habituation of the startle reflex is more sensitive to factors related to the obligatory pathway than to psychological factors.

In terms of understanding the temporal effects of a sustained emotion context on the startle response, a variety of stimulus exposure conditions have been used to manipulate emotional states. Some of these approaches involved increasing exposure time to a single picture (Codispoti, Bradley, \& Lang, 2001; Sutton, Davidson, Donzella, Irwin, \& Dottl, 1997), repetitive presentations of identical pictures (Ferrari, Bradley, Codispoti, \& Lang, 2010), rapid presentations of a series of pictures shown at varying rates (Smith, Löw, Bradley, \& Lang, 2006) as well as examining persistence of emotion effects into the interstimulus interval (Bradley, Cuthbert, \& Lang, 1996). While many of these studies implicate factors such as novelty and attention, limiting generalisability of the results to the specific effects of emotional valence, a prominent finding nonetheless has been that consistently activating a negative emotional state heightens or maintains defensive responses across time.

Using a passive picture viewing paradigm, Smith, Bradley 
and Lang (2005) attempted to separate potential effects of emotion (the psychological pathway) from general habituation effects (the obligatory pathway) by analysing startle magnitudes occurring early and late within three successive blocks of pictures, and then comparing startles during the first block to startles during the last block. Within blocks, they found potentiation of startles occurring later compared to startles occurring earlier for unpleasant pictures, but not for pleasant or neutral pictures, indicating selective potentiation of aversive motivational processes during sustained emotion exposure. When startle responses were compared across rather than within the three blocks however, there was no change in magnitude for unpleasant or neutral pictures, but there was a decrease in startle magnitude for pleasant pictures, indicating selective attenuation of appetitive motivational processes after a longer induction of emotional states. They further suggest that this was not due to habituation of the obligatory pathway since the rate of reduction of the startle magnitude was not consistent across valence conditions.

In the current experiment, rather than assessing the cumulative effects of sustained emotion exposure on the startle response early and late in a block of emotional pictures, the aim was to investigate how startle responses differ during brief, alternating exposure to emotional pictures compared to during sustained exposure. In addition, this study aimed to compare startle responses with online emotional response rating for each picture to see whether any effect of sustained exposure to emotional pictures could be observed in changes in explicit selfreports of emotional responses. This is of special interest, because previous studies demonstrated that some aspects of affective information processing modify startle reflex amplitudes while leaving explicit rating unchanged (Walla et al., 2010; Geiser et al., 2011; Grahl et al., 2012; Walla et al., 2013). This phenomenon gives reason to believe that self reported emotion reflects only a fraction of ongoing raw affective processing (see Walla \& Panksepp, 2013), an idea that deserves more attention than it has previously got.

In this experiment, two modified picture viewing paradigms were used-one in which consistently-valanced stimuli were presented over an extended amount of time and one in which the valence of the presented stimuli was randomised and therefore continually alternating. The design, therefore, involved six response conditions and was analysed using a 2 (Presentation order: Block, Mix) by 3 (Picture valence: Unpleasant, Neutral, Pleasant) within-subjects design. To do this, 90 novel pictures from the IAPS were used, 45 in each paradigm and 15 per valence condition (unpleasant, pleasant and neutral). Pictures from each valence category were chosen so that all categories were matched on intensity of valence and arousal based on pre-evaluations of the IAPS picture collection. Pictures high in arousal were chosen to accentuate valence effects in the startle response (Cuthbert, Bradley, \& Lang, 1996). Self-reported arousal ratings were collected to verify these conditions as well as autonomic physiological arousal indices by recording skin conductance changes. Self-reported pleasantness and arousal ratings were assessed using the Self-Assessment Manikin (SAM), a pictorial rating system accompanying the IAPS collection, which has demonstrated reliability for predicting emotional responses to these picture stimuli, and is widely used in startle research. In addition, mood (The Affect Grid) was assessed prior to the experiment in order to reference ratings to a baseline affective state.

Consistent with previous findings, we expected that picture presentations in the mixed and blocked viewing paradigms would result in emotion-dependent modulation of the startle response magnitude, specifically that startle responses would be largest when viewing unpleasant pictures compared to neutral and smallest when viewing pleasant pictures compared to neutral. Consistent with findings reported by Smith, Bradley and Lang (2005), we expected startle responses to be largest during blocked unpleasant pictures, reflecting a potentiation effect during sustained exposure to unpleasant stimuli. Since current theory holds that the emotion-modulated startle response is an online indicator of an internal emotional state, it was hypothesised that self-reported pleasantness ratings would also reflect the direction of startle magnitude, specifically that pleasantness ratings would be lowest for blocked unpleasant pictures compared to be mixed.

\section{Method}

\section{Ethics Statement}

The ethics committee of the University of Newcastle approved this study (H-2010-1061).

\section{Participants}

The present study is based on 17 consenting participants. They were all undergraduate students of the University of Newcastle (16 females). Mean age was 21.53 years $(\mathrm{SD}=3.48$, range 18 - 29 years). Participants were right-handed in accordance with Oldfield's criteria (Oldfield, 1971), non-smokers with normal hearing and normal or corrected vision and had no history of neuropathology.

\section{Assessments and Measures}

\section{Current Mood}

Ongoing mood can influence decision making behaviour and alter startle baseline reactivity (Grüsser, Wölfling, Mörsen, Kathmann, \& Flor, 2007). Therefore, immediately following the consenting procedure participant's mood was assessed using the Affect Grid (Russell, Weiss, \& Mendelsohn, 1989). The Affect Grid is a single item two dimensional bipolar assessment of pleasure-displeasure and arousal-calm. Users are instructed to place a cross within the two dimensional grid, which is then converted to two individual scores ranging from one to nine for each axis.

\section{Self-Reported Valence and Arousal Ratings}

A computerised version of the Self-Assessment Manikin (SAM); (Bradley \& Lang, 1994) was used to assess affect along valence and arousal dimensions using two nine-point likert scales ( 1 = negative valence or low arousal, respectively; 9 = positive valence or high arousal, respectively). Each SAM scale includes a non-verbal pictorial assessment of a human-like stimulus with visible emotional expressions. Using five figures for each scale, SAM permits the assessment of core valence and arousal dimensions of emotional experience. This is a standard assessment technique for evaluating affective responses for the IAPS collection and is widely used in startle research.

\section{Physiological Measures}

NeXus-10 (produced by Mind Media BV) wireless recording and Bio-trace + software was used to measure and process both 
skin conductance and eye blink responses. Skin conductance was recorded at a rate of 32 samples/s with a Nexus-10-SC/ GSR sensor (two finger sensor) connected to the Nexus-10 recording system with a 24 bit resolution which is able to register changes of less than .0001 microsiemens. We attached one sensor to the middle finger and the other sensor to the ring finger of the left hand.

Eye blinks were elicited by a repeatedly presented startle stimulus (see below for details). With bipolar electromyography (EMG) carried out with the Nexus-10 muscle potential changes of the musculus orbicularis oculi (which elicit eye blinks) of the left eye of every study participant were measured and stored on the hard drive of a lap top computer. We used a dual channel electrode cable with carbon coating and active shielding technology for low noise and an additional ground electrode cable. EMG sampling rate was 2048 per s. A band pass filter from 20 $\mathrm{Hz}$ to $500 \mathrm{~Hz}$ was applied during online recording. Raw EMG data were then recalculated by using the root mean square (RMS) method to transform EMG signals into amplitudes. The resulting amplitudes were then subject to statistical analysis.

\section{Stimuli}

\section{Visual Stimuli}

The emotion eliciting stimuli were 90 pictures (30 neutral, 30 unpleasant and 30 pleasant) from the International Affective Picture System collection (IAPS); (Lang, Bradley, \& Cuthbert, 2005). Pictures were selected based on pre-evaluated ratings of picture pleasantness and arousal. Pictures rated high for valence (unpleasant and pleasant) and arousal (unpleasant, neutral and pleasant) dimensions were selected. An Analysis of Variance (ANOVA) and t-tests were used to confirm respective criteria matched across picture categories. The means and standard deviations of the pictures for this experiment are displayed in Table 1. The pictures were presented in colour against a black background on a computer monitor screen.

\section{Acoustic Startle Stimuli}

Our startle stimulus was a $50 \mathrm{~ms}$ burst of acoustic white noise at $105 \mathrm{~dB}$ sound pressure level delivered through professional headphones fully covering both ears. Sound pressure level was measured with a mobile measuring device (produced by Voltcraft). To achieve the respective loudness a commercial headphone pre-amplifier was used (Behringer; MicroAMP HA400).

\section{Design}

Pictures were randomly allocated to the mixed or blocked

Table 1.

Mean values of visual picture stimuli based on pre-evaluations using the Self Assessment Manikin (SAM) rating system.

\begin{tabular}{ccccc}
\hline & \multicolumn{4}{c}{ Rating } \\
\cline { 2 - 5 } & \multicolumn{2}{c}{ Pleasantness } & \multicolumn{2}{c}{ Arousal } \\
\hline \multirow{2}{*}{ Unpleasant } & 2.65 & .45 & 6.58 & .36 \\
Neutral & 4.52 & .23 & 5.41 & .62 \\
Pleasant & 7.28 & .46 & 6.60 & .38 \\
\hline
\end{tabular}

presentation session using a random number generator. Pictures in the mixed presentation session were further quasi-randomly arranged to ensure constant variation in picture valence. To minimise the potential for order effects, five uniquely randomised lists of pictures were used interchangeably throughout the recruiting period. Hence, each new list contained a different combination of pictures in each viewing session, and all six experimental conditions were fully counterbalanced. Four of the 15 pictures in each valence category were simultaneously associated with a startle-eliciting stimulus at the fifth second of viewing. Pictures associated with the startle-eliciting stimulus were also varied. The interval between two startle-eliciting stimuli was randomised to occur at approximately 35,57 or 79 seconds to prevent learning effects. For both sessions, each picture trial lasted approximately 22 seconds and included a one second cross fixation screen, a six second presentation of the lead picture stimulus, a user-paced SAM pleasantness rating screen and a user-paced SAM arousal rating screen. A black screen of one second duration was used to transition between rating and picture screens.

\section{Procedure}

Experimental testing was conducted within a two month recruiting period at the University of Newcastle Callaghan Campus Functional Neuro-imaging laboratory in individual two hour sessions at regulated temperature $\left(20.5^{\circ} \mathrm{C}\right)$. Consenting participants completed assessments for demographics and pre-experimental affect respectively before being seated in front of a computer monitor and connected to the physiological recording equipment by the experimenter. Participants were instructed to pay close attention to their feelings throughout the session and to quickly rate their immediate reaction to each picture for all trials by using a computer mouse to click on the SAM rating. Participants were also told to ignore any loud bursts of noise through the headphones. Prior to commencing the sessions participants practised using the rating system for three trials including one item of extreme valence from each category (unpleasant and pleasant extremes and one neutral) which served as an anchor for subsequent picture rating. The mixed and blocked sessions were separated by a two minute break interval. Three pilot trials were conducted prior to official data collection.

\section{Data Reduction}

Subjective reports of emotion on trials associated with the acoustic startle may be biased by the inherently startling nature of the probe. Therefore SAM ratings for each valence category were taken from four startle-unassociated trials. Similarly, other physiological measures can be augmented by the startle probe. To avoid this unwanted source of variation data for skin conductance were taken exclusively from the six second picture presentation interval on startle-associated trials for each valence category. Extracted values were then averaged to form mean physiological values for each of the six valence-viewing conditions.

A trigger sound detector was used to identify onset of the acoustic probe in the data read out. This served as a reference for detecting the true onset of startle reactivity. The startle blink value was defined as the peak rise in EMG waveform on startle associated trials. Trials for which peak rise in EMG waveform was less than $5 \mu \mathrm{V}$ were scored as missing. To decrease the 
probability of artifact being mistaken for a true response a trial was scored as rejected if excessive noise occurred. For each participant trial scores were averaged to create a mean value for each valence category.

\section{Statistical Analysis}

Normality checks of the EMG data distribution revealed a unimodal significant positive skew of startle data $(z=8.47, p$ $<.01)$. To partial out floor effects, the EMG distribution was $\log$ transformed prior to inferential statistical analyses. Covariation of the dependent variables Self-reported Pleasantness and Startle Modulation with the variable Current Mood (valence dimension) was assessed by performing respective bivariate correlations. No significant relationship was found therefore current mood was not included as a covariate.

To assess the independent effects of self-reported and startle reflex responses, a repeated measures Analyses of Variance (ANOVA) was carried out for each dependent variable under a 2 (Presentation order: Block, Mix) by 3 (Picture valence: Unpleasant, Neutral, Pleasant) within subjects design. Simple contrasts were used to determine the direction of significant main effects $(p<.05)$. Greenhouse-Gessier corrections for sphericity (if $\epsilon<.75$ ) and Bonferroni corrections were applied where required.

\section{Results}

\section{Emotional Valence Measures}

\section{Self-Reported Pleasantness}

The descriptive statistics for emotional valence measures including pleasantness ratings and startle blink magnitudes are displayed in Table 2. For the main effect of picture valence on self-reported pleasantness Mauchly's test indicated a violation of sphericity, $\chi^{2}=11.12, p=.004, \epsilon=.65$. However, as theory would predict, picture valence had a strongly significant effect on pleasantness ratings in the expected directions ( $F(1.29$,

Table 2.

Mean emotional responses to unpleasant, neutral and pleasant pictures as a function of presentation order.

\begin{tabular}{ccccc}
\hline & \multicolumn{2}{c}{ Mix } & \multicolumn{2}{c}{ Block } \\
\hline Variable & $\mathrm{M}(\mathrm{SE})$ & $95 \% \mathrm{CI}$ & $\mathrm{M}(\mathrm{SE})$ & $95 \% \mathrm{CI}$ \\
\hline $\begin{array}{c}\text { Self-reported } \\
\text { pleasantness }\end{array}$ & & & & \\
Unpleasant & $2.33(.24)$ & {$[1.82,2.84]$} & $2.30(.24)$ & {$[1.78,2.81]$} \\
Neutral & $3.92(.22)$ & {$[3.46,4.39]$} & $3.94(.28)$ & {$[3.35,4.50]$} \\
Pleasant & $6.05(.29)$ & {$[5.44,6.67]$} & $5.86(.27)$ & {$[5.30,6.40]$} \\
Total & $4.10(.15)$ & {$[3.79,4.41]$} & $4.03(.20)$ & {$[3.61,4.46]$} \\
Startle blink reflex & & & & \\
Unpleasant & $3.35(.12)$ & {$[3.11,3.60]$} & $3.13(.14)$ & {$[2.85,3.42]$} \\
Neutral & $3.15(.16)$ & {$[2.81,3.49]$} & $3.15(.16)$ & {$[2.81,3.48]$} \\
Pleasant & $3.11(.11)$ & {$[2.88,3.34]$} & $2.96(.14)$ & {$[2.66,3.25]$} \\
Total & $3.21(.12)$ & {$[2.95,3.46]$} & $3.08(.14)$ & {$[2.79,3.37]$} \\
\hline
\end{tabular}

Note: $\mathrm{M}=$ Mean; $\mathrm{SE}=$ Standard Error; $\mathrm{CI}=$ Confidence Interval. Values represent adjusted mean and parameter estimates. ${ }^{a}$ Startle blink reflex amplitudes $(\mu \mathrm{V})$ represent the natural logarithm of the sample distribution.
19.38) $=80.33, p<.001, \eta^{2}=.84$, after Greenhouse Geisser corrections for sphericity violation). As shown in Figure 1, unpleasant pictures were rated as significantly more unpleasant than neutral pictures $\left(F(1,15)=99.2, p<.001, \eta^{2}=.87\right)$ and pleasant pictures $\left(F(1,15)=100.6, p<.001, \eta^{2}=.87\right)$, while pleasant pictures were rated as significantly more pleasant than neutral pictures $\left(F(1,15)=45.31, p<.001, \eta^{2}=.75\right)$.

Neither the main effect of presentation order, nor its interaction with picture valence resulted in significant augmentation of ratings $\left(F(1,15)=.18, p=.67, \eta^{2}=.01 ; F(2,30)=.25, p\right.$ $=.78, \eta^{2}=.02$, respectively). Non-significant tests were however, associated with low observed power $(7 \%$ and $9 \%$, respectively).

\section{Startle Blink Reflex}

Figure 2 shows the mean startle reflex magnitudes for each picture viewing condition. For the main effect of picture valence on startle blink magnitude Mauchly's test indicated a violation of sphericity $\left(\chi^{2}=6.55, p=.038, \epsilon=.74\right)$, however there was a moderately significant main effect $(F(1.48,23.64)=4.77, p$ $=.027, \eta^{2}=.23$, after Greenhouse Geisser corrections for

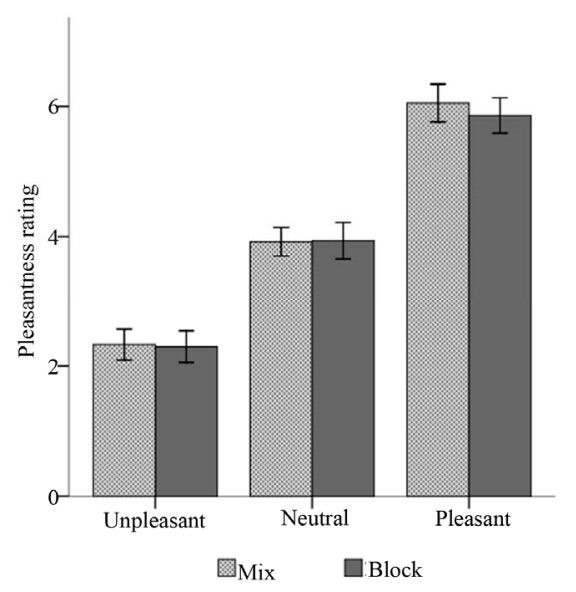

Figure 1.

Bar graph representing mean self-reported pleasantness ratings and standard error bars as a function of picture viewing conditions.

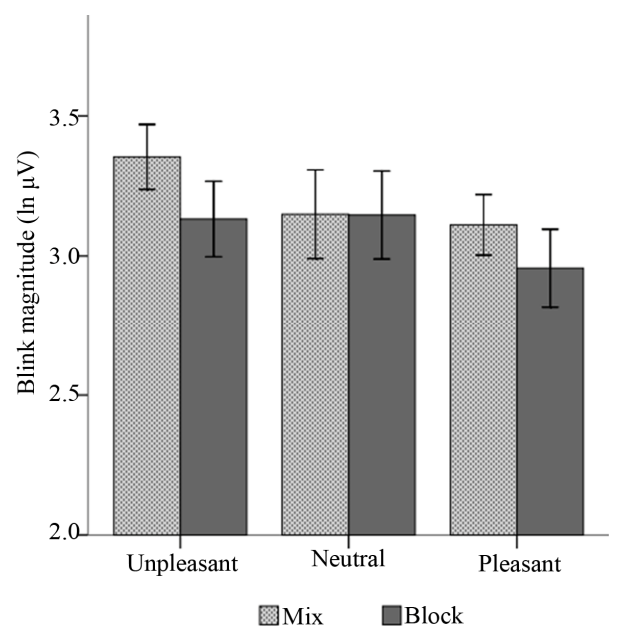

Figure 2.

Bar graph representing mean self-reported arousal ratings and standard error bars as a function of picture viewing conditions. 
sphericity violation). Despite sphericity violations, the main ANOVA was used to interpret significant effects due to the small sample size and low power associated with multivariate analyses. Contrasts revealed that mean startle responses to unpleasant pictures were significantly potentiated compared to those for pleasant pictures $\left(F(1,16)=13.94, p=.002, \eta^{2}=.47\right)$, with high observed power for detecting a small effect (94\%), but not compared to those for neutral pictures $(F(1,16)=1.24$, $\left.p=.283, \eta^{2}=.07\right)$. However, observed power was low $(18 \%)$. Mean startle responses to pleasant pictures were reduced compared to neutral pictures, and the difference was approaching significance $\left(F(1,16)=3.91, p=.065, \eta^{2}=.20\right)$, with moderate power for detecting an effect $(46 \%)$.

The main effect of presentation order was marginally significant $\left(F(1,16)=4.68, p=.046, \eta^{2}=.23\right)$ with moderate observed power $(53 \%)$, and corresponds to an overall reduction in mean startle responses to blocked picture presentations. The mean effect size of blocked order $(-.13 \ln \mu \mathrm{V})$ corresponded to a large effect $(F=.54)$. Together, power and effect size estimates suggest low probability of type two errors.

The interaction of presentation order and picture valence was not significant $F(2,32)=2.31, p=.115$, however two paired sample t-tests were run to further evaluate the relationship between variables of interest (corrected $p=.025$ ). Although approaching significance, the mean magnitude of startle responses during blocked unpleasant pictures were not significantly reduced compared to those during mixed unpleasant pictures $(t(16)=2.3, p=.035)$. The mean magnitude of startle responses during blocked pleasant pictures were also not significantly reduced compared to those during mixed pleasant pictures, $t(16)=$ $1.73, p=.103$.

\section{Emotional Arousal Measures}

\section{Self-Reported Arousal}

Descriptive statistics for arousal measures including arousal ratings and skin conductance are included in Table $\mathbf{3}$. There was a main effect of picture valence on self-reported arousal ratings $\left(F(2,30)=13.45, p<.001, \eta^{2}=.47\right.$; Figure 3$)$. Contrasts revealed that unpleasant pictures were significantly more arousing than both pleasant $\left(F(1,15)=15.62, p<.001, \eta^{2}\right.$ $=.51)$ and neutral pictures $\left(F(1,15)=18.8, p=.001, \eta^{2}=.56\right)$, and corresponds to high observed power $(96 \%$ and $98 \%$, respectively). Pleasant pictures were no more arousing than neutral pictures $\left(F(1,15)=.98, p=.338, \eta^{2}=.06\right)$. There was however low observed test power for detecting a significant effect of picture valence on arousal (15\%).

There was no main effect of presentation order on self-reported arousal $\left(F(1,15)=.26, p=.62, \eta^{2}=.02\right)$, nor was there an interaction between presentation order and picture valence $\left(F(2,30)=.63, p=.54, \eta^{2}=.04\right)$. These effects also correspond to low observed test power ( $8 \%$ and $15 \%$, respectively).

\section{Skin Conductance}

There was no main effect of picture valence on skin conductance $\left(F(2,30)=.08, p=.92, \eta^{2}=.01\right)$, however there was a significant main effect of presentation order, $F(1,15)=8.3, p$ $=.011, \eta^{2}=.36$ (Figure 4). Blocked picture presentations elicited marginally lower skin conductance responses $(M=3.14$, $\mathrm{SE}=.27, \mathrm{CI}=2.57,3.72)$ than did mixed presentations $(\mathrm{M}=$ $3.24, \mathrm{SE}=.27, \mathrm{CI}=2.67,3.81)$. The mean effect of blocked order $(-.01 \mu \mathrm{S})$ was large $(F=.75)$ and corresponds to acceptable
Table 3.

Mean arousal responses to unpleasant, neutral and pleasant pictures as a function of presentation order.

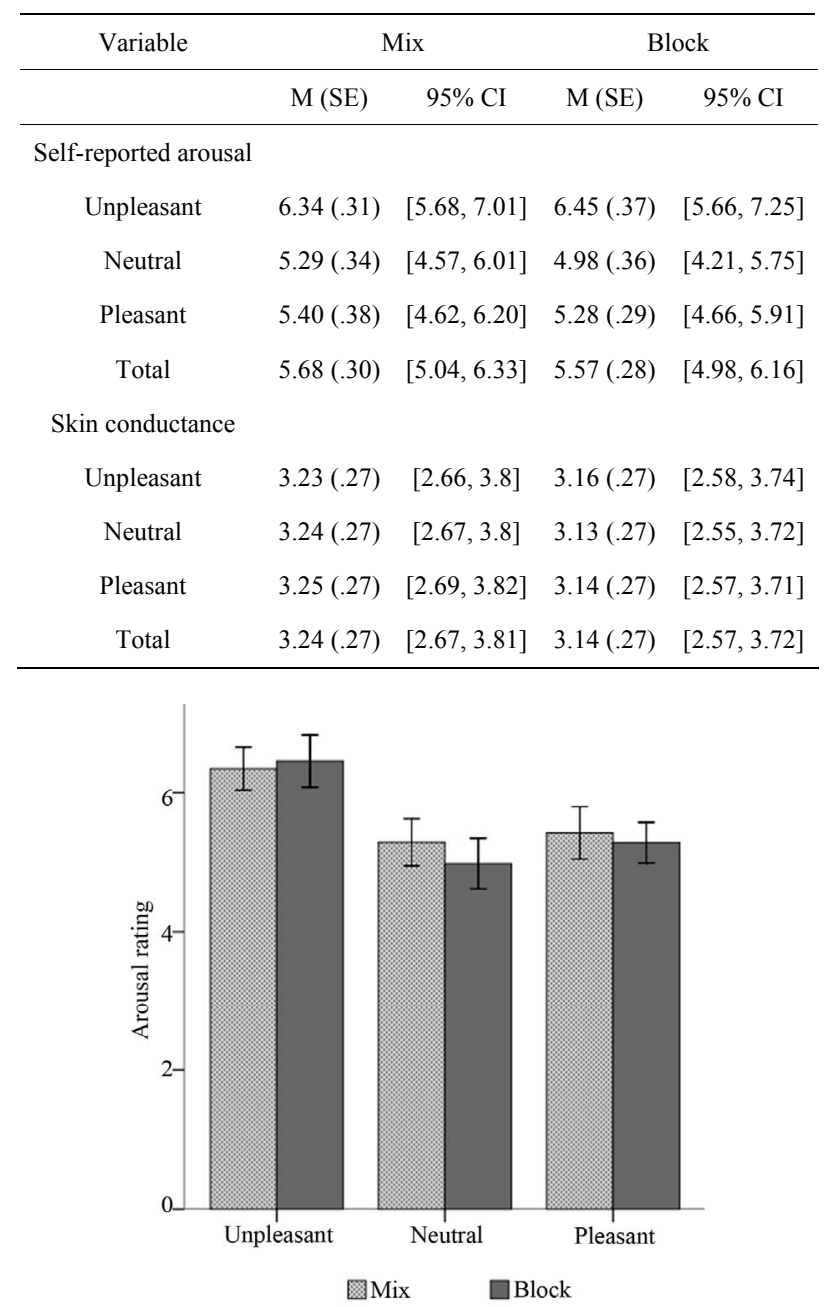

Figure 3.

Bar graph representing mean self-reported arousal ratings and standard error bars as a function of picture viewing conditions.

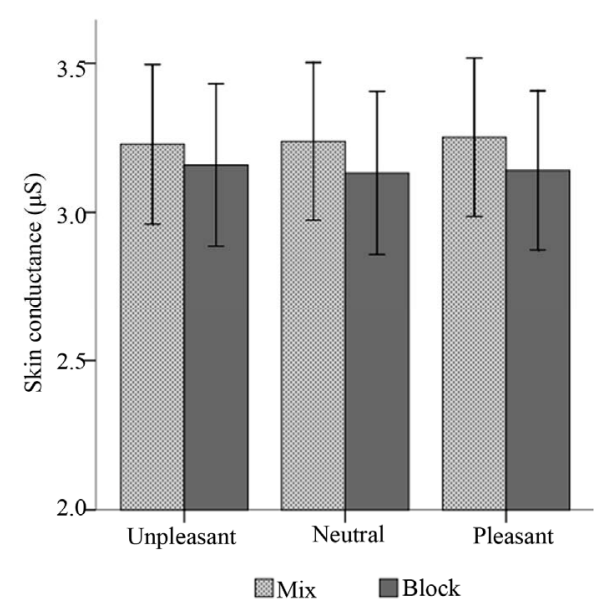

Figure 4.

Bar graph representing mean skin conductance levels and standard error bars as a function of picture viewing conditions. 
observed power $(77 \%)$. There was no interaction between presentation order and picture valence $\left(F(2,30)=.67, p=.52, \eta^{2}\right.$ $=.04)$.

Three paired sample $\mathrm{t}$ tests (corrected $p=.017$ ) were carried out on variables of interest. The t-tests revealed that mean skin conductance levels were significantly reduced during blocked compared to mixed neutral pictures $(t(15)=2.83, p=.013)$, but not during blocked compared to mixed pleasant $(t(15)=2.45, p$ $=.027)$ or unpleasant $(t(15)=1.88, p=.08)$ pictures.

\section{Discussion}

The question addressed in the current study was whether emotional responses are differently affected by sustained exposure to emotional stimuli compared to brief exposure, as measured by self-reported emotional responses and the affective startleblink reflex. Previous research using a passive picture viewing paradigm has demonstrated that cumulative exposure can potentiate startles during negative conditions (Smith et al., 2005). Therefore, it was hypothesised that sustained exposure to unpleasant emotional pictures would potentiate startle responses. It was further hypothesised that self-reported emotional responses would reflect these changes in startle responses. The results do not support either hypothesis, and instead, suggest that sustained exposure to emotional pictures caused a general attenuation of startle magnitudes across all valence conditions. In addition, this effect was not observed in selfreported emotional responses, which instead showed no difference in ratings across brief and sustained emotion exposure conditions.

This study assessed the effects of sustained exposure to emotional stimuli while also recording immediate self-reported emotional reactions to the presented stimuli. This procedure differs to the procedure used by Smith, Bradley and Lang (2005) where participants were required to passively view emotional pictures without any additional rating task. Recently, Wangelin, Low, McTeague, Bradley, and Lang (2011) investigated the effects of a cognitive distractor task on startle responses during sustained aversive exposure. They found that embedded distractor tasks did eliminate potentiation of startles during sustained exposure to unpleasant pictures compared to when pictures were viewed passively. This suggests that sustained effects of emotion on the startle reflex are quite sensitive to cognitive and perceptual distractions. This is also strengthened by additional data from an earlier experiment (Bradley et al., 1996) which showed that startle responses were maintained over time when a passive picture viewing task was combined with a concurrent auditory-based emotion word recognition task (which was used to ensure participants paid attention to the acoustic startle stimuli). Therefore a possible explanation for why startles were not potentiated as expected during unpleasant picture viewing was that the rating task itself inadvertently acted as a distraction, momentarily diverting attention from the sustained emotion, eliminating or downgrading cumulative effects. However, to draw any conclusions in this area would require a deliberate comparison of startle responses to "actively" and "passively" viewed pictures in a single experiment.

Alternatively, research examining perceptual differences related to different emotional tasks has found differences in neural activity extending to limbic regions as a function of task instructions such rating an emotional reaction compared to recognising a specific emotion (e.g. Liberzon et al., 2000). Therefore, use of a rating task in the current study may have differently affected perceptual processing and subsequent motivational system activation compared to other studies of sustained emotion that used a recognition task or a simple passive viewing task. Said differently, it is possible that actively paying attention to ones own emotions rather than simply observing emotional events can have a regulatory influence, impeding the formation of a stronger negative emotional state. Walla et al. (2013a) found that self referenced negative emotion images were rated more negative that un-referenced negative images and self-referenced positive images were rated more positive than un-referenced positive images. In their study, startle responses were enhanced as a result of self reference regardless of emotion valence. This finding was interpreted as being reflective of motivation as indicated by startle reflex modulation data. Thus, the notion arises that in the current study too motivation is reflected in the startle reflex modulation data and motivation is reduced over time if emotion valence is kept constant over longer periods of time.

A second important finding was that contrary to what would be predicted by past empirical investigations, startle responses elicited across all unpleasant pictures were not significantly potentiated relative to neutral, nor were startle responses during pleasant pictures significantly reduced relative to neutral. This raises concern as to whether the lead stimuli used in this experiment were effective for inducing the desired emotional states. One possible explanation is that the trend towards attenuated startle responses may have washed out the potentiation effects for unpleasant pictures. However, this does not explain the null effect produced by pleasant pictures, which may alternatively be the result of lower than expected arousal levels associated with pleasant pictures, which is apparent in the low self-reports of arousal for pleasant pictures. It should be noted that most of our participants were female and most of the pleasant pictures from the IAPS involve erotica, which are generally rated as more pleasant by males than females. Thus, these unexpected findings were most likely caused by a gender bias in the sample. However, it should also be noted that the IAPS collection has to be treated with caution, because some of the images that are pre-evaluated as neutral may elicit affective valence, perhaps depending on cultural background. After all, startle reflex modulation has been repeatedly shown to be sensitive to raw affective processing in a variety of experiments using many different lead stimuli. Urban neighbourhoods (Geiser et al., 2011), food intake (Walla et al., 2010), bottle shape (Grahl et al., 2012) and brand attitude (Walla et al., 2011) were shown to significantly modulate startle responses.

A third and incidental finding was differences between selfreported arousal ratings and skin conductance levels. While there was no effect of mixed and blocked order on self-reported arousal ratings, skin conductance was reduced during blocked presentations, with post hoc tests revealing that the effect was only significant for neutral pictures. Habituation of skin conductance levels over time has previously been documented by Bradley, Lang, \& Cuthbert (1993), who also documented the relationship between arousal and the startle response (Cuthbert et al., 1996), showing that changes in arousal moderate the affective startle response such that a high level of arousal is necessary for valence-modulated differences to take effect. Therefore, it is likely that despite efforts to control for arousal across valence and order conditions, reduced startle response magnitudes seen in the current study were simply the result of habituated arousal levels.

It should still be noted that several functional MRI studies have documented habituation of amygdala responses during re- 
peated exposure to negative emotional stimuli such as pictures of fearful faces (Wright et al., 2001) and other unpleasant pictures (Liberzon et al., 2000). Zald (2003) reviewed studies of amygdala activation patterns during emotional evaluations of visual stimuli and concluded that habituation "may reflect a process through which stimuli are rapidly re-evaluated based on the lack of consequences arising from their previous exposure." (Zald, 2003: p. 104). Given that the amygdala is a region implicated in the startle neural circuitry (Yeomans \& Frankland, 1995), it is possible that reduced startle responses may be reflecting activation related to amygdala circuitry.

\section{Conclusion}

Several important implications for emotion research have arisen from the current experiment. Firstly, neural pathways involved in the affective modulation of the startle response may be more sensitive to emotion exposure than can be observed via self-reported emotional responses. Hence, although the startle reflex is able to predict general directional differences in emotional states, a closer investigation suggests it is, at least in part, measuring emotion processing activity that is somewhat different to and inaccessible by self-reports. Second, this study provides conflicting results compared to other studies investigating the effect of sustained exposure to emotional pictures, and suggests that certain conditions of picture viewing, specifically, active emotional rating compared to passive viewing, may differently affect the way emotional stimuli are processed. This finding may be relevant for research directed at understanding or developing cognitive strategies for optimised and adaptive emotional perception and comprehension, and may also be an important factor to consider when deciding how to best design emotion experiments. Finally, the findings of this study may have useful implications for clinical populations (see ArthurKelly et al., 2013) as well as for advancing consumer neuroscience (Koller \& Walla, 2012; Walla et al., 2013b).

\section{REFERENCES}

Arthur-Kelly, M., Lyons, G. S., \& Walla, P. (2013). Toward improved ways of knowing children with Profound Multiple Disabilities (PMD): Introducing startle reflex modulation. Developmental Neurohabilitation, in press.

Bradley, M., Cuthbert, B., \& Lang, P. (1996). Picture media and emotion: Effects of a sustained affective context. Psychophysiology, 33, 662-670. doi:10.1111/j.1469-8986.1996.tb02362.x

Bradley, M., \& Lang, P. (1994). Measuring emotion: The self-assessment manikin and the semantic differential. Journal of Behavior Therapy and Experimental Psychiatry, 25, 49-59. doi:10.1016/0005-7916(94)90063-9

Bradley, M., Lang, P., \& Cuthbert, B. (1993). Emotion, novelty, and the startle reflex: Habituation in humans. Behavioral Neuroscience, 107, 970-980. doi:10.1037/0735-7044.107.6.970

Codispoti, M., Bradley, M. M., \& Lang, P. J. (2001). Affective reactions to briefly presented pictures. Psychophysiology, 38, 474-478. doi:10.1111/1469-8986.3830474

Cuthbert, B., Bradley, M., \& Lang, P. (1996). Probing picture perception: Activation and emotion. Psychophysiology, 33, 103-111. doi:10.1111/j.1469-8986.1996.tb02114.x

Ferrari, V., Bradley, M., Codispoti, M., \& Lang, P. (2010). Repetitive exposure: Brain and reflex measures of emotion and attention. Psychophysiology, 47, 1-8.

Filion, D., Dawson, M., \& Schell, A. (1998). The psychological significance of human startle eyeblink modification: A review. Biological Psychology, 47, 1-43.

doi:10.1016/S0301-0511(97)00020-3
Geiser, M., \& Walla, P. (2011). Objective measures of emotion during virtual walks through urban environments. Applied Sciences, 1, 1-11.

Grahl, A., Greiner, U., \& Walla, P. (2012). Bottle-shape elicits genderspecific emotion: A startle reflex modulation study. Psychology, 3, 548-554. doi:10.4236/psych.2012.37081

Grüsser, S., Wölfling, K., Mörsen, C., Kathmann, N., \& Flor, H. (2007). The influence of current mood on affective startle modulation. Experimental Brain Research, 177, 122-128. doi:10.1007/s00221-006-0653-x

Koller, M., \& Walla, P. (2012). Measuring affective information processing in information systems and consumer research-introducing startle reflex modulation. ICIS Proceedings, 33rd International Conference on Information Systems, Orlando, FL. http://aisel.aisnet.org/icis2012/proceedings/BreakthroughIdeas/1/

Lang, P., Bradley, M., \& Cuthbert, B. (2005). International affective picture system (IAPS): Instruction manual and affective ratings. Gainesville, FL: The Center for Research in Psychophysiology, University of Florida.

Liberzon, I., Taylor, S. F., Fig, L. M., Decker, L. R., Koeppe, R. A., \& Minoshima, S. (2000). Limbic Activation and Psychophysiologic Responses to Aversive Visual Stimuli: Interaction with Cognitive Task. Neuropsychopharmacology, 23, 508-516. doi:10.1016/S0893-133X(00)00157-3

Oldfield, R. (1971). The assessment and analysis of handedness: The Edinburgh inventory. Neuropsychologia, 9, 97-113. doi:10.1016/0028-3932(71)90067-4

Russell, J., Weiss, A., \& Mendelsohn, G. (1989). Affect grid: A singleitem scale of pleasure and arousal. Journal of Personality and Social Psychology, 57, 493-502. doi:10.1037/0022-3514.57.3.493

Smith, J. C., Bradley, M., \& Lang, P. (2005). State anxiety and affective physiology: Effects of sustained exposure to affective pictures. Biological Psychology, 69, 247-260. doi:10.1016/j.biopsycho.2004.09.001

Smith, J. C., Löw, A., Bradley, M. M., \& Lang, P. J. (2006). Rapid picture presentation and affective engagement. Emotion, 6, 208. doi: $10.1037 / 1528-3542.6 .2 .208$

Sutton, S., Davidson, R., Donzella, B., Irwin, W., \& Dottl, D. (1997). Manipulating affective state using extended picture presentations. Psychophysiology, 34, 217. doi:10.1111/j.1469-8986.1997.tb02135.x

Walla, P., Rosser, L., Scharfenberger, J., Duregger, C., \& Bosshard, S. (2013a). Emotion ownership: Different effects on explicit ratings and implicit responses. Psychology, 4, 213-216.

Walla, P., Richter, M., Färber, S., Leodolter, U., \& Bauer, H. (2010). Food evoked changes in humans: Startle response modulation and Event-Related Potentials (ERPs). Journal of Psychophysiology, 24, 25-32. doi:10.1027/0269-8803/a000003

Walla, P., \& Panksepp, J. (2013). Neuroimaging helps to clarify brain affective processing without necessarily clarifying emotions, novel frontiers of advanced neuroimaging.

http://www.intechopen.com/books/novel-frontiers-of-advanced-neur oiming/neuroimaging-helps-to-clarify-brain-affective-processing-without-necessarily-clarifying-emotions doi:10.5772/51761

Walla, P., Mavratzakis, A., \& Bosshard, S. (2013b). Neuroimaging for the affective brain sciences, and its role in advancing consumer neuroscience, novel frontiers of advanced neuroimaging.

http://www.intechopen.com/books/novel-frontiers-of-advanced-neur oimaing/neuroimaging-for-the-affective-brain-sciences-and-its-rolein-advancing-consumer-neuroscience doi:10.5772/51042

Wangelin, B. C., Low, A., McTeague, L. M., Bradley, M. M., \& Lang, P. J. (2011). Aversive picture processing: Effects of a concurrent task on sustained defensive system engagement. Psychophysiology, 48, 112-116. doi:10.1111/j.1469-8986.2010.01041.x

Wright, C., Fischer, H., Whalen, P., McInerney, S., Shin, L., \& Rauch, S. (2001). Differential prefrontal cortex and amygdala habituation to repeatedly presented emotional stimuli. Neuroreport, 12, 379. doi: 10.1097/00001756-200102120-00039

Yeomans, J., \& Frankland, P. (1995). The acoustic startle reflex: Neurons and connections. Brain Research Reviews, 21, 301-314. doi:10.1016/0165-0173(96)00004-5

Zald, D. (2003). The human amygdala and the emotional evaluation of sensory stimuli. Brain Research Reviews, 41, 88-123. doi:10.1016/S0165-0173(02)00248-5 